\title{
Prognostic Impact of Histologic Subtype in Non-Small Cell Lung Cancer Patients Treated with Gamma Knife Radiosurgery: Retrospective Analysis of 104 Patients
}

\author{
Fatih AYDEMIR ${ }^{1}$, Kadir TUFAN ${ }^{1}$, Melih CEKINMEZ ${ }^{1}$, Ozgur KARDES ${ }^{1}$, Feyzi Birol SARICA ${ }^{1}$, Erkan TOPKAN², \\ Erkin SONMEZ ${ }^{3}$, Ozlem ALKAN ${ }^{4}$, Gamze UGURLUER ${ }^{5}$, Nur ALTINORS ${ }^{3}$ \\ 'Baskent University, School of Medicine, Adana Training and Research Center, Department of Neurosurgery, Adana, Turkey \\ ${ }^{2}$ Baskent University, School of Medicine, Adana Training and Research Center, Department of Radiation Oncology, Adana, Turkey \\ ${ }^{3}$ Baskent University, School of Medicine, Ankara Training and Research Center, Department of Neurosurgery, Ankara, Turkey \\ ${ }^{4}$ Baskent University, School of Medicine, Adana Training and Research Center, Department of Radiology, Adana, Turkey \\ ${ }^{5}$ Acıbadem University, School of Medicine, Adana Training and Research Center, Department of Radiation Oncology, Adana, Turkey
}

\section{ABSTRACT}

AIM: In this study, factors affecting survival, local failure, distant brain failure, whole brain failure and whole-brain radiation therapy (WBRT) free survival according to histological subtypes were investigated in patients with brain metastases from non-small cell lung cancer (NSCLC).

MATERIAL and METHODS: Patients with positive pathology reports for adenocarcinoma (ACA) and squamous cell carcinoma (SCC) were included in the study. Seventy-eight ACA and 26 SCC patients were included in the study. Patients with previous history of cerebral metastasis surgery and WBRT were excluded from the study.

RESULTS: The median survival was calculated as 12.6 months for patients with ACA and 5.9 months for patients with SCC. One-year distant brain failure was calculated as $65.1 \%$ in ACA patients and $39.6 \%$ in SCC patients. One-year whole brain failure was calculated as $58.1 \%$ in ACA patients and $39.6 \%$ in SCC patients. The one-year freedom from WBRT rate was calculated as $72.8 \%$ in ACA patients and $56.3 \%$ in SCC patients. SCC histology was considered as a significant factor in deterioration of overall survival in multivariate analysis. SCC histology, the increase in the number of metastases and RPA class were factors that caused an increase in distant brain failure. Also, SCC histology, the increase in the number of metastases and RPA class were factors that caused an increase in whole brain failure.

CONCLUSION: SCC histology may be an important prognostic factor for overall survival. Also, due to high distant brain failure rate in SCC histology, WBRT can be added to treatment early.

KEYWORDS: Cranial metastases, Gamma knife radiosurgery, Non-small cell lung cancer

\section{INTRODUCTION}

$\mathrm{B}$ rain metastases (BM) have been reported in $40 \%$ of patients with cancer (15). The factors that determine treatment options in patients with brain metastasis are age, Karnofsky Performance Scale (KPS), primary tumor histopathology, radiosensitivity, number of intracranial metastasis, and location. The brain has been reported to be the most common region for metastasis in patients with lung cancer, with a rate of $20-50 \%$. Treatment options include whole-brain radiation therapy (WBRT), stereotactic radiosurgery (SRS), surgery, or a combination treatment (4). The effect of application of SRS alone or in combination with WBRT on brain metastasis 
has been investigated in several studies. In a study published by Aoyama et al., it was reported that although addition of WBRT to the treatment regime of patients who had undergone SRS provided a reduction in relapses, it was not effective in lengthening average survival times (2). Adding WBRT to the treatment regime of patients with one to three brain metastases treated with SRS or surgery decreased intracranial recurrence and neurological mortality. However, improvement in functional independence and mean survival time has not been determined (10). The most important problem with WBRT is that it is associated with worsening neurocognitive functions. Chang et al. found that the risk of decrease in learning and memory functions in the first four months after treatment is higher in patients treated with SRS and WBRT than in patients treated only with SRS (7). In a study published by Tsao et al., it was reported that control of local and distant brain metastases in patients treated with only SRS was less successful compared to patients treated with SRS and WBRT (19).

In recent studies, the histological subtype of the primary tumor has been emphasized to be important in the natural course of brain metastases. Histological subtypes of lung cancer are known to behave differently in the natural course of the disease. Small cell lung cancer (SCLC) has a high rate of brain metastasis and prophylactic cranial irradiation is the standard treatment in these patients $(3,17)$. In addition, survival of adenocarcinoma (ACA), a histological subtype of non-small cell lung cancer (NSCLC), is known to be better compared to squamous cell carcinoma (SCC) (6). Studies on the effects of different histological subtypes of NSCLC on the biological behavior of the brain metastases have increased recently.

In this study, we report retrospective examination of ACA and SCC patients who were treated with Gamma Knife Radiosurgery (GKRS) by a similar protocol in a single center and who did not receive therapeutic WBRT for diagnosed brain metastases. Factors affecting survival, local failure, distant brain failure, whole brain failure, and duration of life without WBRT were investigated.

\section{MATERIAL and METHODS}

\section{Study Design}

The study was approved by the institutional review board of Baskent University before collection of patient information, and was conducted according to the principles of the Declaration of Helsinki and the rules of Good Clinical Practice (Project no: KA14/353). The medical records of patients treated with GKRS for brain metastases due to lung cancer in the Neurosurgery Department of Baskent University, Adana Training and Research Hospital between June 2011 and December 2014 were reviewed retrospectively. Patients with positive pathology reports for ACA and SCC were included in the study. Patients with previous history of cerebral metastasis surgery and WBRT were excluded from the study. Patient data were obtained from the electronic medical records system. Tumor volume and dosimetric data were obtained from the Gamma Plan treatment planning system (Gamma plan 10.0) archives.

\section{Data Collection}

Age, gender, histopathology, survival, control of primary disease, extracranial metastatic disease, Recursive Partitioning Analysis (RPA) class, time to cranial metastases after diagnosis of lung carcinoma, number of metastases, GKRS dose, local-distant and whole brain control, WBRT after gamma knife, WBRT-free time, and neurological death were recorded for patient data. Survival was calculated from the date of application of GKRS. Local, distant, and whole brain control period was calculated as the time from the date of application of GKRS to the time of local, distant, and whole brain failure (i.e., the date of the last radiological imaging in the absence of local, distant, and whole brain failure). WBRT-free time was calculated as the time from the date of application of GKRS to the date of application of GKRS and was equal to survival in WBRT-untreated patients. Recursive partitioning analysis was identified according to the analysis report by Gaspar et al. (8).

\section{Radiosurgery Technique}

Patients were treated on a Leksell Model 4C Unit (Elekta, Stockholm, Sweden). Before radiosurgery, the patients underwent a high-resolution contrast-enhanced stereotactic magnetic resonance imaging (MRI) study of the brain. Treatment planning was performed using the Leksell GammaPlan Treatment Planning System (Gamma plan 10.0). A median dose of 20 Gray (Gy; range: $14-24$ Gy) was generally prescribed to the $50 \%$ isodose line at the margin of each metastasis. Dose prescription was based on guidelines published by Shaw et al. (16). Patients were treated one to four weeks after the detection of brain metastasis. A total of 200 tumors in 104 patients and a median of one lesion (range: 1-6) per patient were treated. Median tumor volume was $3.72 \mathrm{~cm}^{3}$ (range: 0.13-49.02 $\mathrm{cm}^{3}$ ) in all patients, $2.72 \mathrm{~cm}^{3}$ (range: $0.13-40.21 \mathrm{~cm}^{3}$ ) in ACA patients, and $8.82 \mathrm{~cm}^{3}$ (range: $0.23-49.02 \mathrm{~cm}^{3}$ ) in SCC patients. Total tumor volumes were greater than $30 \mathrm{~cm}^{3}$ in only four patients: two in SCC and two in ACA histology, respectively.

\section{Patient Follow-Up, Toxicity, Salvage Therapy}

Patients were followed by serial MRls, first at the eighth week after GKRS treatment and following ones with 3-month periods. The median follow-up time of the patients was 8.8 months (mean: 10.7 months, range: 1.3-36.8 months). Local failure was defined by a combination of clinical and radiological signs of local treatment failure. Patients with suspected local treatment failure were generally followed initially with short interval imaging and were treated conservatively before determination of a treatment failure to rule out radiation necrosis. Local failures were treated with surgical excision, GKRS, or observation. In the case of local failure, we performed a cranial MRI for four consecutive weeks. If we defined progression of the tumor, we applied reGKRS. If we defined Grade 3 radionecrosis, surgical excision was performed. Distant brain progression was defined by the occurrence of new metastases that were not visible initially. Distant brain failures were generally treated with GKRS, and WBRT was generally reserved for four or more total brain metastases over time or for short-interval distant failures. 
Whole brain failures were defined as failure to achieve local or distant control. Death due to intracranial progression was defined as "death due to specifically progressive neurological impairment with stable systemic disease" (14). Four patients developed Grade 3 radionecrosis and surgical excision was performed. GKRS was re-applied to 12 patients in whom local or distant failure was seen. $28.2 \%$ (22/76) of ACA patients and $42.3 \%(11 / 26)$ of SCC patients underwent WBRT.

\section{Statistical Methods}

Statistical analyses were performed based on patient stratification according to BM histology: ACA versus SCC. Frequency distributions were used to describe categorical variables, and mean, median, and ranges were used for quantitative variables. Demographic features were compared between the ACA and SCC cohorts using a Chi-square test. Overall survival, local tumor control, distant brain failure, whole brain failure, and WBRT-free survival of patients were calculated using Kaplan-Meier analysis. Survival times were calculated as the time between the first day of GKRS and the date of death/last visit for OS; the date of local or inbrain relapse, or the date of death/last visit for local tumor control; and distant brain failure time, and the time between the first day GKRS and the first day of WBRT for WBRT-free survival, respectively. Survival analysis was performed by the Kaplan-Meier method and the survival curves of subsets were compared with two-sided log-rank tests. Only the factors that exhibited statistical significance in univariate analysis were included in multivariate analysis that utilized Cox proportional hazards regression tests. Multivariate analyzes were performed for each survival end-point (i.e., overall survival, distant brain failure, whole brain failure, and WBRTfree survival). All tests were two-tailed, and a $p$-value $<0.05$ was considered significant.

\section{RESULTS}

\section{Patients Characteristics}

78 ACA and 26 SCC patients were included in the study. $87.5 \%$ of the patients were male and $12.5 \%$ were female. The median age of all patients was 60 years (range: $35-86$ years); median age was 59.5 years (range: $35-86$ years) for ACA patients and 65.5 years (range: $47-83$ years) for SCC patients. Median time from diagnosis of lung cancer to diagnosis of brain metastasis was 2.5 months (range: 0.0-48.0 months) for all patients; it was one month (range: 0-48) for ACA patients and seven months (range: 0-34 months) for SCC patients. Extracranial metastatic disease was identified in $47.4 \%$ of ACA patients and $57.2 \%$ of SCC patients. $12.5 \%$ of patients were RPA Class $1,73.1 \%$ were RPA Class 2, and $14.4 \%$ were RPA Class 3. Patient characteristics are summarized in Table I.

\section{Univariate Analysis}

The Kaplan-Meier method was used to determine the effects of histology, age, gender, number of metastases, RPA class, volume, and extracranial metastases factors on overall survival, local tumor control, distant brain control, whole brain control, and WBRT-free survival. The number of metastases was classified as one or greater than one. Volume was classified as above and below $3.72 \mathrm{~cm}^{3}$, which is the average median volume of all patients. Univariate $p$-value is shown in Table II.

\section{Survival}

The median overall survival for all patients was 8.5 months (95\% Cl: 3.8-13.2), whereas the one-year overall survival rate was $47.1 \%$, and the two-year overall survival rate was $10.2 \%$. According to the histopathology of the patients, the overall median survival was 12.6 months (95\% Cl: 8.8-16.4) for ACA patients and 5.9 months $(95 \% \mathrm{Cl}: 4.4-7.4)$ for SCC patients $(p=.024)$. One- and two-year overall survival rates were calculated as $53.8 \%$ and $12.8 \%$ in ACA patients and $26.9 \%$ and $0.0 \%$ in SCC patients, respectively. The Kaplan-Meier estimates of overall survival are shown in Figure 1. The median overall survival according to RPA classification was calculated as 14.3 months (95\% Cl: $11.8-16.8)$ for Class $1,10.3$ months (95\% Cl: $5.7-14.9)$ for Class 2, and 3.5 months $(95 \% \mathrm{Cl}$ : 2.5-4.5) for Class $3 \quad(p<.0001)$. Kaplan-Meier estimates for overall survival stratified by RPA Class are found in Figure 2. Overall median survival of patients without extracranial metastases was 14.1 months (95\% Cl: 12.9-15.3), whereas it was 4.9 months (95\% Cl: $4-5.7)$ in patients with extracranial metastases $(p<.0001)$. Kaplan-Meier estimates for overall survival stratified by extracranial metastases are found in Figure 3. The effects of age, gender, volume, and the number of metastases on overall survival could not be demonstrated. 95 of the 104 patients died during the analysis. The cause of death could not be determined in 16 patients. Death due to intracranial progression occurred in $23.1 \%(18 / 76)$ of ACA and $30.8 \%(8 / 26)$ of SCC patients who died.

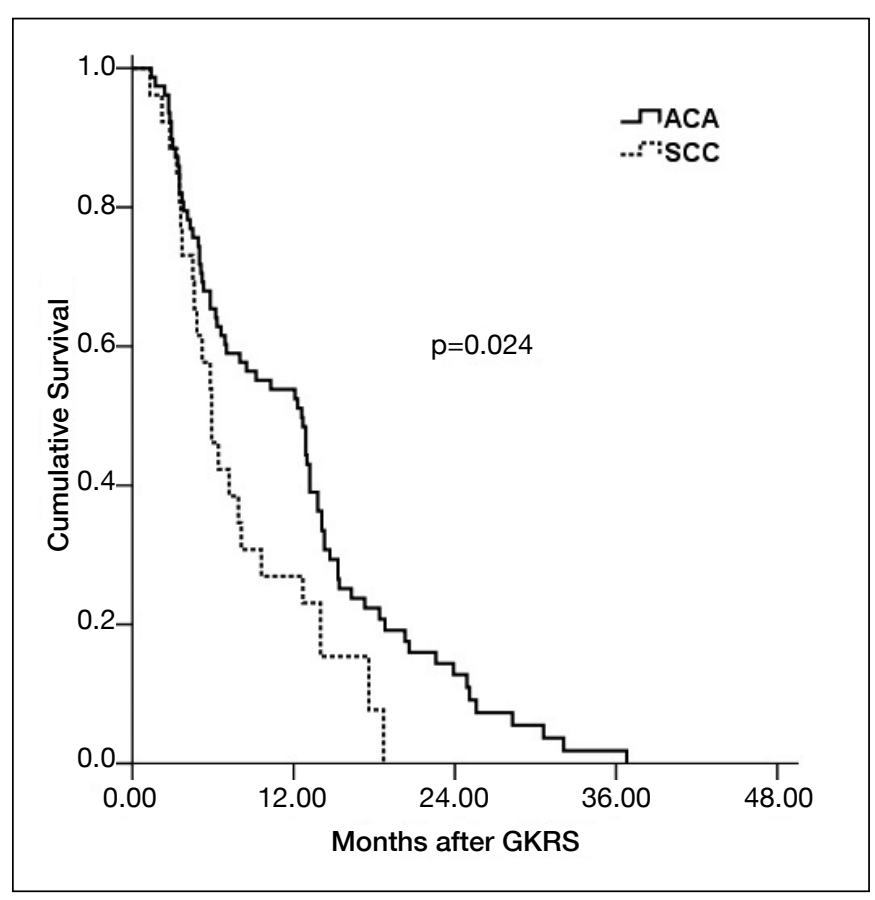

Figure 1: Kaplan-Meier plot of overall survival. (ACA, adenocarcinoma; SCC, squamous cell carcinoma). 


\section{Local, Distant, and Whole Brain Failure}

Median time of freedom from local failure was 22.5 months (95\% Cl: 10.6-34.4) for all patients, whereas one- and two-year freedom from local failure was $87.0 \%$ and $41.4 \%$, respectively. Median time of freedom from local failure in ACA patients was 22.5 months, whereas it was 12.7 months $(95 \% \mathrm{Cl}$ : $12.0-$ 13.3) in SCC patients $(p=.116)$. One- and two-year freedom from local failure was calculated as $86.6 \%$ and $45.6 \%$ in ACA patients and $87.5 \%$ and $36.5 \%$ in SCC patients, respectively. The effects of age, gender, RPA class, extracranial metastatic disease, and number of metastases on local control could not be demonstrated statistically.

Median time of freedom from distant brain failure was 14.3 months (95\% Cl: $10.6-18.0)$ for all patients, whereas one- and two-year freedom from distant brain failure was $58.5 \%$ and $21.7 \%$, respectively. Median time of freedom from distant brain failure in ACA patients was 15.7 months (95\% Cl: 12.7-18.7), whereas it was 5.7 months $(95 \% \mathrm{Cl}$ : 4.3-7) in SCC patients $(p=.006)$. One and two-year freedom from distant brain failure was calculated as $65.1 \%$ and $25.3 \%$ in ACA patients and $39.6 \%$ and $19.8 \%$ in SCC patients, respectively. KaplanMeier estimates for freedom from distant failure stratified by histology are found in Figure 4. RPA class $(p=<.0001)$, presence of extracranial metastatic disease $(p=.005)$ and the increase in the number of metastases $(p=.001)$ all reduce distant control. The effects of age, gender, and volume on distant control could not be demonstrated statistically.

Median time of freedom from whole brain failure was12.4 months (95\% Cl: 8.8-16) for all patients, whereas one- and two-year freedom from whole brain failure was calculated as $53.2 \%$ and $8 \%$, respectively. Median time of freedom from whole brain failure in ACA patients was 13.2 months $(95 \% \mathrm{Cl}$ : 11.3-15), whereas it was 5.7 months (95\% Cl: 4.3-7) in SCC patients $(p=.013)$. One and two-year freedom from whole brain failure was calculated as $58.1 \%$ and $10.6 \%$ in ACA patients and $39.6 \%$ and $10.4 \%$ in SCC patients, respectively. KaplanMeier estimates for freedom from whole-brain failure stratified

Table I: Patient Characteristics

\begin{tabular}{|c|c|c|c|c|}
\hline Characteristic & $\begin{array}{c}\text { Total } \\
(n=104)\end{array}$ & $\begin{array}{c}\text { ACA } \\
(n=78)\end{array}$ & $\begin{array}{c}\text { SCC } \\
(n=26)\end{array}$ & p-value \\
\hline $\begin{array}{l}\text { Age; years } \\
\text { (range) }\end{array}$ & $\begin{array}{l}60 \\
(35-86)\end{array}$ & $\begin{array}{l}59.5 \\
(35-86)\end{array}$ & $\begin{array}{l}65.5 \\
(47-83)\end{array}$ & 0.17 \\
\hline \multicolumn{5}{|l|}{ Gender no. (\%) } \\
\hline Male & $91(87.5)$ & 67 (85.9) & $24(92.3)$ & 0.93 \\
\hline Female & $13(12.5)$ & $11(14.1)$ & $2(7.7)$ & \\
\hline \multicolumn{5}{|l|}{ RPA Class no.(\%) } \\
\hline 1 & $13(12.5)$ & $9(11.5)$ & $4(15.4)$ & \\
\hline 2 & $76(73.1)$ & $59(75.6)$ & $17(65.4)$ & 0.36 \\
\hline 3 & $15(14.4)$ & $10(12.8)$ & 5 (19.2) & \\
\hline \multicolumn{5}{|l|}{ Lesions treated, no. (\%) } \\
\hline 1 & $57(54.8)$ & $40(51.3)$ & $17(65.4)$ & \multirow{4}{*}{0.23} \\
\hline 2 & $23(22.1)$ & $19(24.4)$ & $4(15.4)$ & \\
\hline 3 & $14(13.5)$ & $11(14.1)$ & $3(11.5)$ & \\
\hline$\geq 4$ & $10(9.6)$ & $8(10.2)$ & $2(7.7)$ & \\
\hline Median tumor volume; $\left(\mathrm{cm}^{3}\right)$ & $\begin{array}{c}3.72 \\
(0.13-49.02)\end{array}$ & $\begin{array}{c}2.72 \\
(0.13-40.21)\end{array}$ & $\begin{array}{c}8.82 \\
(0.23-49.02)\end{array}$ & 0.01 \\
\hline Median marginal dose, Gy (range) & $\begin{array}{l}20 \\
(14-24)\end{array}$ & $\begin{array}{l}20 \\
(14-24)\end{array}$ & $\begin{array}{l}20 \\
(14-24)\end{array}$ & 1.00 \\
\hline \multicolumn{5}{|l|}{ WBRT status (\%) } \\
\hline Yes & $33(31.7)$ & $22(28.2)$ & $11(42.3)$ & \multirow{2}{*}{0.86} \\
\hline No & $71(68.3)$ & $56(71.2)$ & $15(57.7)$ & \\
\hline Local failures (\%) & $13(12.5)$ & $9(11.5)$ & $4(15.4)$ & 0.57 \\
\hline Distant failures (\%) & $39(37.5)$ & $26(34.6)$ & $13(50)$ & 0.08 \\
\hline Whole brain failures (\%) & $46(44.2)$ & $31(39.7)$ & $15(57.7)$ & 0.03 \\
\hline Deaths due to intracranial progression (\%) & $26(25)$ & $18(23.1)$ & $8(30.8)$ & 0.18 \\
\hline Extracranial metastasis (\%) & $52(50)$ & $37(47.4)$ & $15(57.2)$ & 0.25 \\
\hline
\end{tabular}

RPA: Recursive partitioning analysis, WBRT: Whole-brain radiation therapy. 
Table II: Univariate Analysis of Possible Predictive Factors for Overall Survival, Local, Distant, Whole Brain Control and Freedom from WBRT

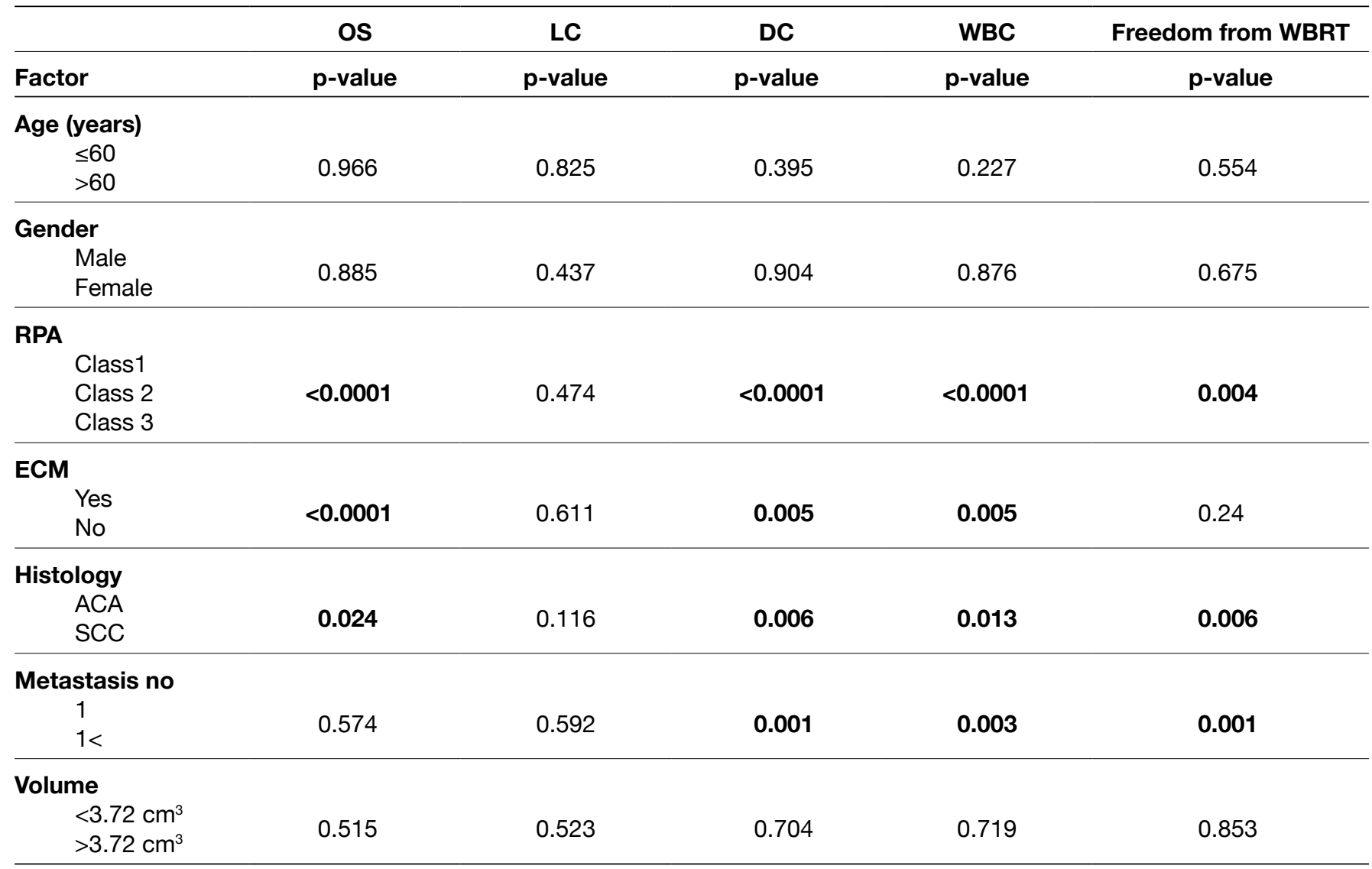

OS: Overall survival, LC: Local control, DC: Distant control, WBC: Whole-brain control, WBRT: Whole-brain radiation therapy, RPA: Recursive partitioning analysis, ECM: Extracranial metastases, ACA: Adenocarcinoma; SCC: Squamous cell carcinoma.

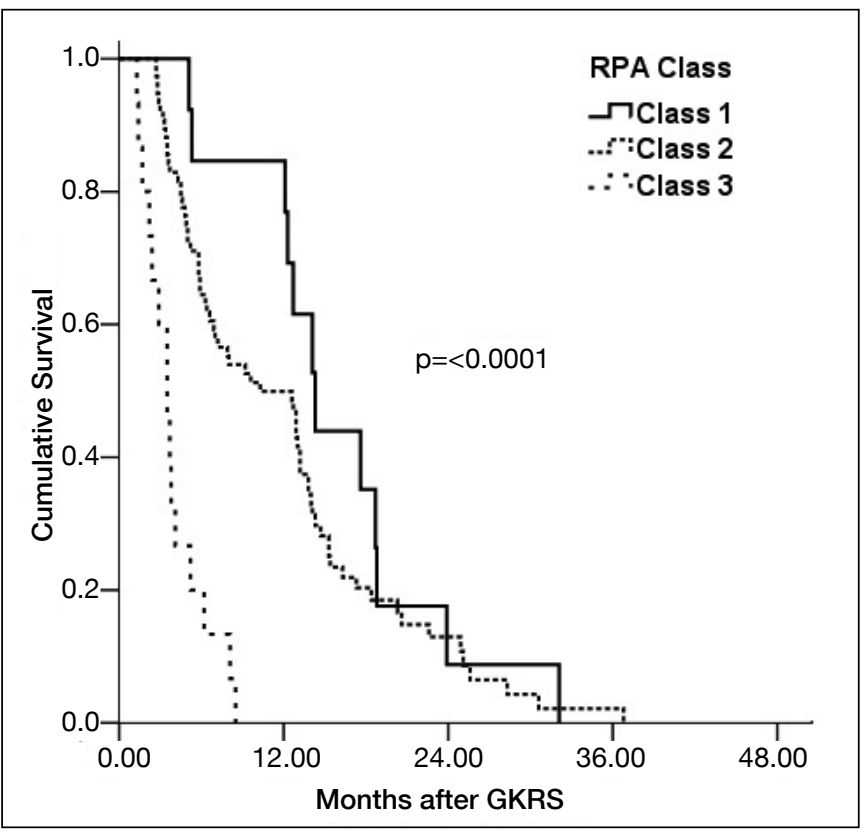

Figure 2: Kaplan-Meier plot of overall survival. (RPA: Recursive partitioning analysis).

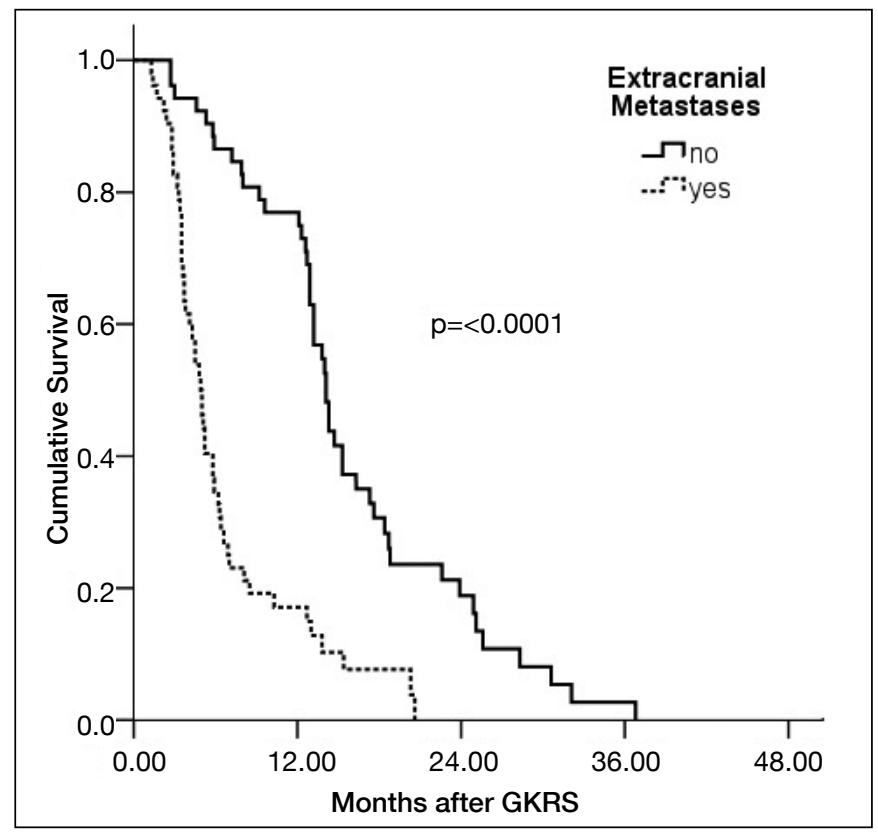

Figure 3: Kaplan-Meier plot of overall survival. 
by histology are found in Figure 5. RPA class $(p=<.0001)$, presence of extracranial metastatic disease $(p=.005)$, and increase in the number of metastases $(p=.003)$ all reduce whole brain control. The effects of age, gender, and volume on whole brain control could not be demonstrated statistically.

\section{Time Delay to WBRT}

Median time of freedom from WBRT was 17.8 months $(95 \% \mathrm{Cl}$ : 13.3-22.3) for all patients, whereas one- and two-year freedom from WBRT was calculated as $68.3 \%$ and $34.5 \%$, respectively. Median time to application of WBRT in ACA patients was 18.6 months (95\% Cl: 11.4-25.8), whereas it was 12.9 months (95\% Cl: $0.0-26.2)$ in SCC patients $(p=.006)$. The one- and two-year freedom from WBRT rate was calculated as $72.8 \%$ and $39.9 \%$ in ACA patients and $56.3 \%$ and $21.1 \%$ in SCC patients, respectively (Figure 6). While RPA class $(p=.004)$ and increase in the number of metastases $(p=.001)$ reduced time of freedom from WBRT, the effects of age, gender, volume, and presence of extracranial metastatic disease on freedom from WBRT could not be demonstrated statistically.

\section{Multivariate Analysis}

Multivariate analyses were performed using Cox proportional hazards regression testing. Factors that impacted overall survival, distant brain failure, whole brain failure, and WBRTfree survival were evaluated. In multivariate analysis, SCC histology was considered as a significant factor on the deterioration of overall survival $(p=.017)$. RPA class $(p=.017)$ and extracranial metastasis $(p=<.0001)$ were important factors that cause deterioration in overall survival. SCC histology $(p=.001)$, increase in the number of metastases $(p=.004)$, and RPA class $(p=.004)$ were factors that caused an increase in distant brain failure. Also, SCC histology $(p=.001)$, increase in the number of metastases $(p=.009)$ and RPA class $(p=<.0001)$

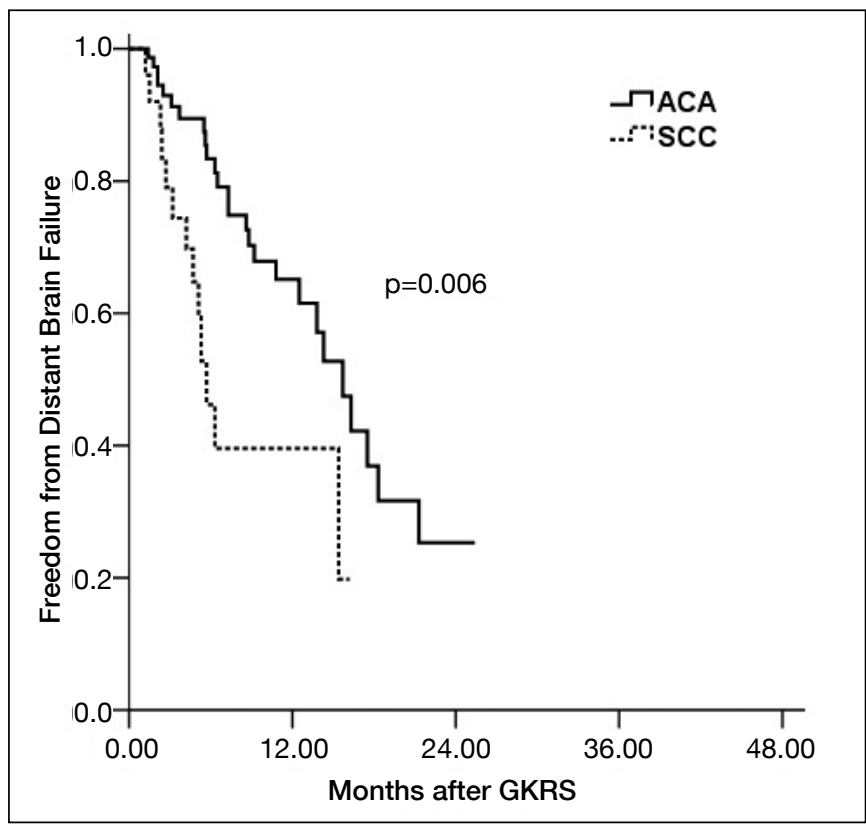

Figure 4: Kaplan-Meier plot of distant control. (ACA, adenocarcinoma; SCC, squamous cell carcinoma). were factors that caused an increase in whole brain failure. Although the effect of extracranial metastatic disease on distant control and whole brain control was shown in univariate analysis, no statistically significant effect was found in the multivariate analysis. SCC histology $(p=.001)$ and number of metastases $(p=.002)$ were factors that shortened WBRT-free time. RPA class $(p=.052)$ tended to affect. The results of the multivariate analysis with associated $p$-values are shown in Table III.

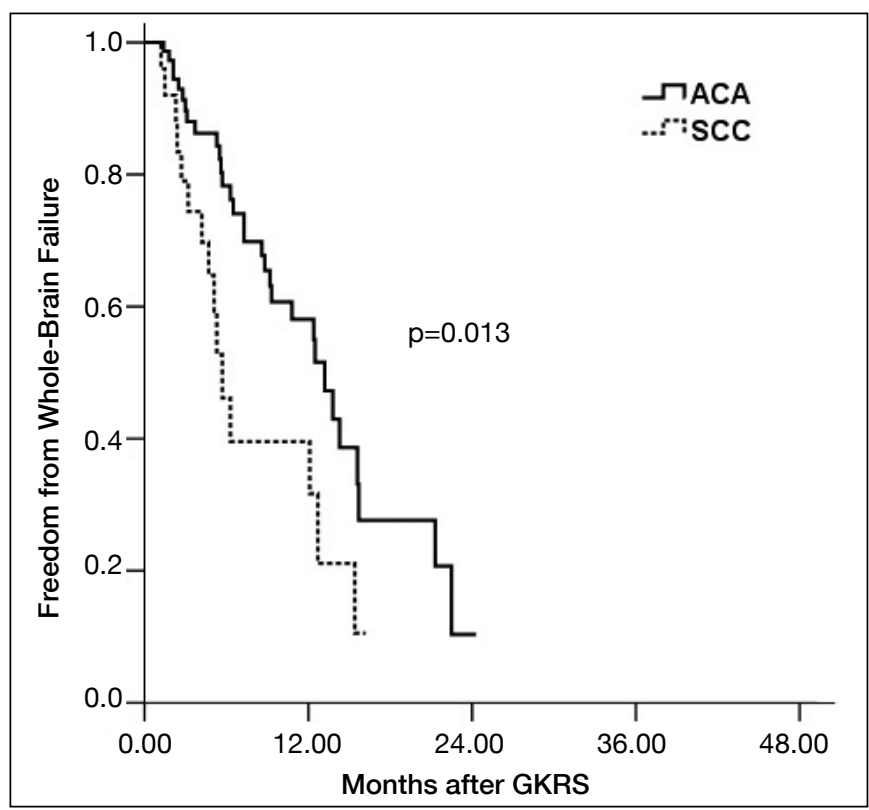

Figure 5: Kaplan-Meier plot of whole-brain control. (ACA adenocarcinoma; SCC, squamous cell carcinoma).

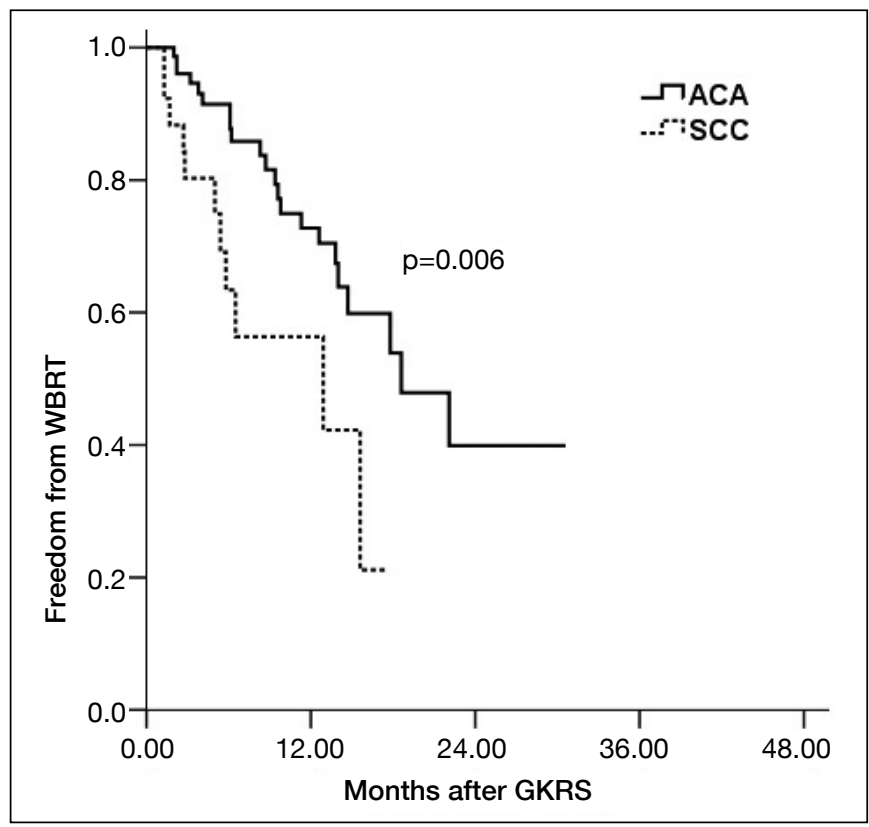

Figure 6: Kaplan-Meier plot of time to whole-brain radiation therapy. (ACA, adenocarcinoma; SCC, squamous cell carcinoma). 


\section{DISCUSSION}

Declines in quality of life and brain metastases that cause death due to intracranial progression have encouraged researchers to find new therapeutic strategies. Several randomized trials have shown that local control and overall survival of patients treated with surgical excision and subsequent WBRT are better than outcomes for patients treated with WBRT alone $(13,14,20)$. As a result, local control has been seen to be effective in overall survival and neurological status, so strategies to improve local control have been developed. Due to surgical complications and surgery causing delay in the treatment of primary disease, interest in radiosurgery has increased recently. Many studies also have shown that SRS combined with WBRT is superior in local control and overall survival compared to WBRT alone $(1,11)$.

Lung cancers usually cause brain metastases, and the literature has generally treated these as non-small cell and small cell cancer. Metastases of SCLC are known to have low local control rates and high rates of death due to intracranial progression. The mechanism for decreased local control in SCLC is unclear. However, populations of radioresistant cells, clonogens, and increased invasion into the brain parenchyma have been proposed as possible mechanisms. Thus, prophylactic WBRT became the standard for managing SCLC $(5,9)$. KPS, age, number of metastases, and presence of extracranial metastases were reported as the dominant factors affecting survival after the diagnosis of brain metastases of lung cancer (18). In our study, RPA class, extracranial metastatic disease and histology were identified as important factors for overall survival. According to our study, RPA Class 3 patients have a median survival of only 3.5 months and GKRS treatment for this group of patients should be questioned.
In the literature, few studies have shown the effect of NSCLC histological subtypes on survival. In RTOG 9508 study, NSCLC patients were grouped according to histological subtypes and survival of SCC after WBRT was found to be worse than in ACA (1).

In a published series of 213 patients who did not receive therapeutic WBRT, Kuremsky et al. compared ACA, SCC, and SCLC cases (12). One-year survival of ACA patients was $56 \%$ as opposed to $20 \%$ in SCC patients; in consideration of these differences in survival rates, the authors stated that histological subtype may represent a new variable between current prognostic indexes. They hypothesized there were two reasons for the difference between ACA and SCC survival rates. These reasons included later relative diagnosis of SCC and better success in systemic therapy of ACA compared to SCC in recent years were thought (12). However, Zairi et al. could not find a statistically significant difference between the results of ACA and SCC in a series of 89 cases (21). In our study, one-year survival of patients with ACA was $56.8 \%$, while it was $26.9 \%$ in SCC patients. This result suggests that histology may be an important factor in survival.

The risk of distant brain failure is known to increase in patients without primary tumor control. In our study, RPA class, large number of metastases, and extracranial metastases increased the risk of distant brain failure. Additionally, the distant brain failure rate in SCC patients was higher than the rate in ACA patients, and this increased the rate of WBRT application. Early addition of WBRT to treatment besides radio surgery should be considered in patients with SCC. Distant brain failure rate is low in ACA patients and WBRT can be used as a salvage regime.

This study had some limitations. As this is a retrospective study and was limited in the number of patients, the results

Table III: Multivariate Analysis of Possible Predictive Factors for Overall Survival, Local, Distant, Whole Brain Control and Freedom from WBRT

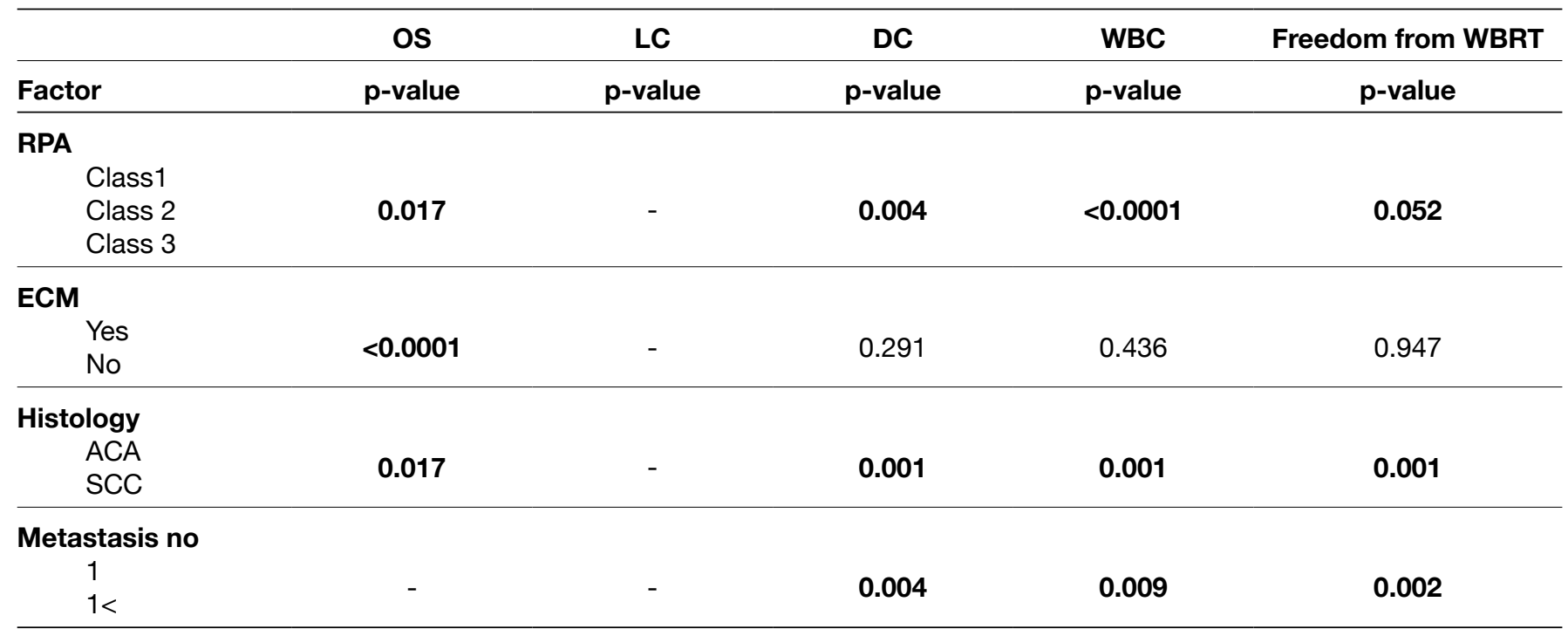

OS: Overall survival, LC: Local control, DC: Distant control, WBC: Whole-brain control, WBRT: Whole-brain radiation therapy, RPA: Recursive partitioning analysis, ECM: Extracranial metastases, ACA: Adenocarcinoma, SCC: Squamous cell carcinoma. 
may have unexpected sources of bias. Although the number of patients diagnosed with SCC was statistically significant, the relatively small number of the patients decreased the statistical power of the study. In further studies, histological subtype should be considered an important prognostic factor.

\section{CONCLUSION}

KPS, age, number of metastases, and presence of extracranial metastases are known factors that affect the overall survival in brain metastases of lung cancer. Our study suggests that SCC histology might also be an important factor for overall survival. In addition, WBRT may be added to treatment early in SCC due to the high rate of distant brain failure. We believe that specific studies that further investigate the impact of histological subtypes will contribute to the treatment protocol.

\section{REFERENCES}

1. Andrews DW, Scott CB, Sperduto PW, Flanders AE, Gaspar LE, Schell MC, Werner-Wasik M, Demas W, Ryu J, Bahary JP, Souhami L, Rotman M, Mehta MP, Curran WJ Jr: Whole brain radiation therapy with or without stereotactic radiosurgery boost for patients with one to three brain metastases: Phase III results of the RTOG9508 randomized trial. Lancet 363:16651672, 2004

2. Aoyama H, Shirato $\mathrm{H}$, Tago $M$, Nakagawa $\mathrm{K}$, Toyoda $\mathrm{T}$, Hatano K, Kenjyo M, Oya N, Hirota S, Shioura H, Kunieda E, Inomata T, Hayakawa K, Katoh N, Kobashi G: Stereotactic radiosurgery plus whole-brain radiation therapy vs. stereotactic radiosurgery alone for treatment of brain metastases: A randomized controlled trial. JAMA 295:2483-2491, 2006

3. Aupérin A, Arriagada R, Pignon JP, Le Péchoux C, Gregor A, Stephens RJ, Kristjansen PE, Johnson BE, Ueoka H, Wagner $\mathrm{H}$, Aisner J: Prophylactic cranial irradiation for patients with small-cell lung cancer in complete remission. Prophylactic Cranial Irradiation Overview Collaborative Group. N Engl J Med 341:476-484,1999

4. Badiyan SN, Ferraro DJ, Yaddanapudi S, Drzymala RE, Lee AY, Silver SA, Dyk P, DeWees T, Simpson JR, Rich KM, Robinson CG: Impact of time of day on outcomes after stereotactic radiosurgery for non-small cell lung cancer brain metastases. Cancer 119:3563-3569, 2013

5. Baumert BG, Rutten I, Dehing-Oberije C, Twijnstra A, Dirx MJ, Debougnoux-Huppertz RM, Lambin P, Kubat B: A pathologybased substrate for target definition in radiosurgery of brain metastases. Int J Radiat Oncol Biol Phys 66:187-194, 2006

6. Bradley JD, Paulus R, Komaki R, Masters GA, Forster K, Schild SE: Randomized phase III comparison of standarddose (60 Gy) versus high-dose (74 Gy) conformal chemoradiotherapy $1 / 2$ cetuximab for stage IIIA/IIIB non-small cell lung cancer: Preliminary findings on radiation dose in RTOG 0617. Int J Radiat Oncol Biol Phys, 81:S2, 2011

7. Chang EL, Wefel JS, Hess KR, Allen PK, Lang FF, Kornguth DG, Arbuckle RB, Swint JM, Shiu AS, Maor MH, Meyers CA: Neurocognition in patients with brain metastases treated with radiosurgery or radiosurgery plus whole-brain irradiation: $A$ randomised controlled trial. Lancet Oncol 10:1037-1044, 2009

8. Gaspar LE, Scott C, Murray K, Curran W: Validation of the RTOG recursive partitioning analysis (RPA) classification for brain metastases. Int J Radiat Oncol Biol Phys 47:1001-1006, 2000
9. Harris S, Chan MD, Lovato JF, Ellis TL, Tatter SB, Bourland JD, Munley MT, deGuzman AF, Shaw EG, Urbanic JJ, McMullen KP: Gamma knife stereotactic radiosurgery as salvage therapy after failure of whole-brain radiotherapy in patients with smallcell lung cancer. Int J Radiat Oncol Biol Phys 83:53-59, 2012

10. Kocher M, Soffietti R, Abacioglu U, Villà S, Fauchon F, Baumert BG, Fariselli L, Tzuk-Shina T, Kortmann RD, Carrie C, Ben Hassel M, Kouri M, Valeinis E, van den Berge D, Collette S, Collette L, Mueller RP: Adjuvant whole-brain radiotherapy versus observation after radiosurgery or surgical resection of one to three cerebral metastases: Results of the EORTC 22952-26001 study. J Clin Oncol 29:134-141, 2011

11. Kondziolka D, Patel A, Lunsford LD, Kassam A, Flickinger JC: Stereotactic radio-surgery plus whole brain radiotherapy versus radiotherapy alone for patients with multiple brain metastases. Int J Radiat Oncol Biol Phys 45:427-434, 1999

12. Kuremsky JG, Urbanic JJ, Petty WJ, Lovato JF, Bourland JD, Tatter SB, Ellis TL, McMullen KP, Shaw EG, Chan MD: Tumor histology predicts patterns of failure and survival in patients with brain metas-tases from lung cancer treated with Gamma Knife Radiosurgery. Neurosurgery 73:641-647, 2013

13. Patchell RA, Tibbs PA, Walsh JW, Dempsey RJ, Maruyama Y, Kryscio RJ, Markesbery WR, Macdonald JS, Young B: A randomized trial of surgery in the treatment of single metatases to the brain. N Engl J Med 322:494-500, 1990

14. Patchell RA, Tibbs PA, Regine WF, Dempsey RJ, Mohiuddin M, Kryscio RJ, Markesbery WR, Foon KA, Young B: Postoperative radiotherapy in the treatment of single metastases to the brain: A randomized trial. JAMA 280:1485-1489, 1998

15. Patchell RA: The management of brain metastases. Cancer Treat Rev 29:533-540, 2003

16. Shaw E, Scott C, Souhami L, Dinapoli R, Kline R, Loeffler J, Farnan N: Single dose radiosurgical treatment of recurrent previously irradiated primary brain tumors and brain metastases: Final report of RTOG protocol 90-05. Int J Radiat Oncol Biol Phys 47:291-298, 2000

17. Slotman B, Faivre-Finn C, Kramer G, Rankin E, Snee M, Hatton M, Postmus P, Collette L, Musat E, Senan S: Prophylactic cranial irradiation in extensive small-cell lung cancer. $\mathrm{N}$ Engl $\mathrm{J}$ Med 357:664-672, 2007

18. Sperduto PW, Kased N, Roberge D, Xu Z, Shanley R, Luo $X$, Sneed PK, Chao ST, Weil RJ, Suh J, Bhatt A, Jensen AW, Brown PD, Shih HA, Kirkpatrick J, Gaspar LE, Fiveash JB, Chiang V, Knisely JP, Sperduto CM, Lin N, Mehta M: Summary report on the graded prognostic assessment: An accurate and facile diagnosis-specific tool to estimate survival for patients with brain metastases. J Clin Oncol 30:419-425, 2012

19. Tsao M, Xu W, Sahgal A: A meta-analysis evaluating stereotactic radiosurgery, whole-brain radiotherapy, or both for patients presenting with a limited number of brain metastases. Cancer 118:2486-2493, 2012

20. Vecht CJ, Haaxma-Reiche H, Noordijk EM, Padberg GW, Voormolen JH, Hoekstra FH, Tans JT, Lambooij N, Metsaars JA, Wattendorff AR, Brand R, Hermans J: Treatment of single brain metastasis: Radiotherapy alone or combined with neuro-surgery? Ann Neurol 33:583-590, 1993

21. Zairi F, Ouammou Y, Le Rhun E, Aboukais R, Blond S, Vermandel M, Deken V, Devos P, Reyns N: Relevance of gamma knife radiosurgery alone for the treatment of non-small cell lung cancer brain metastases. Clin Neurol Neurosurg 125:87-93, 2014 